\title{
Reheating constraints on Kähler Moduli Inflation
}

\author{
R. Kabira,*, A. Mukherjee ${ }^{\mathrm{a},}$, D. Lohiya ${ }^{\mathrm{a}}$, \\ ${ }^{a}$ Department of Physics and Astrophysics, University of Delhi, Delhi-110007, India
}

\begin{abstract}
The end of inflation is connected to the standard cosmological scenario through reheating. During reheating, the inflaton oscillates around the minimum of the potential and thus decays into the daughter particles that populate the Universe at later times. Using cosmological evolution for observable CMB scales from the time of Hubble crossing to the present time, we translate the constraint on the spectral index $n_{s}$ from Planck data to the constraint on the reheating scenario in the context of Kähler Moduli Inflation. We find that the details of the potential is irrelevant if the analysis is done strictly within the slow-roll formalism. In addition, we extend the de-facto analysis generally done only for the pivot scale to all the observable scales which crossed the Hubble radius during inflation. We study how the maximum number of e-folds varies for different scales, and the effect of the equation of state and potential parameters.
\end{abstract}

\section{Introduction}

Inflation is regarded as the standard cosmological paradigm to describe the physics of the very early Universe which tackles several problems in cosmology, particularly the question of the origin of cosmological structures. It leads to a causal mechanism to generate almost scale invariant fluctuations on cosmological scales, with small deviations that follow from the precise microphysics of inflation. This prediction is consistent with the measurements of the cosmic microwave background (CMB) anisotropies by the Planck satellite. The latest data allow us to constrain the inflationary model besides giving a slightly red tilted spectral index $n_{s}=0.9682 \pm$ 0.0062 , ruling out exact scale invariance $n_{s}=1$ at over $5 \sigma$ [1, 2].

In the evolution of the Universe, reheating is a transition epoch connecting the end of inflation to radiation and subsequently matter dominated stages of the Universe. Reheating is important as it is the responsible mechanism for the origin of matter in the Universe [3]. During reheating the vacuum energy of the inflaton field gets transferred to the decay particles. Reheating models

\footnotetext{
* Corresponding author

Email addresses: rakesh.kabir@nic.in (R. Kabir), am@physics.du.ac.in (A. Mukherjee), dlohiya@physics.du.ac.in (D. Lohiya)
}

are classified broadly in two classes, perturbative and non-perturbative. In simple models, Perturbative decay involves a three-point interaction term of the form $\sigma \phi \chi^{2}$ where $\phi, \chi$ and $\sigma$ are the inflaton, some (scalar) decay product and coupling constant with dimensions of mass, respectively 4]. Non-perturbative models include parametric resonance decay [5, 6], tachyonic instability [7 11], and instant preheating 12 .

Just after the inflation ends, the Universe is in the non-thermal preheating state 4. Over time the distribution of states are covered by scattering events. This creates a blackbody spectrum characterized by a final temperature $T_{\text {re }}$, at which the radiation-dominated era begins.

To study reheating in an inflationary scenario for some given interactions between the inflaton and other matter fields, an effective equation of state (eos) has to be derived numerically. In general, the eos is a function of time, $w_{\text {re }}(t)$, and changes as the Universe evolves during the various stages of reheating. During this evolution $w_{\text {re }}(t)$ takes values from $-1 / 3$ at the end of inflation to 0 in the initial phase to $1 / 3$ at the start of the radiation dominance. The densities at the end of inflation and at the end of reheating are related by

$$
\ln \frac{\rho_{\mathrm{re}}}{\rho_{\text {end }}}=-3 \int_{a_{\text {end }}}^{a_{\text {re }}}\left(1+w_{\mathrm{re}}\right) \frac{d a}{a} .
$$

Since the analytic integration of the right hand 
side may not be possible for the arbitrary time dependent $w_{\text {re }}$, it is often considered to be constant throughout the entire reheating era. However in the recent literature, time-varying eos has also been considered [13] (also see [14] for a related analysis).

As the fluctuations generated during reheating remain sub-horizon, they cannot leave an observable imprint at the level of the CMB or LSS [15. Therefore it is difficult to constrain the reheating era, except in some speculative scenarios [16 26]. Still we can put lower and upper bounds on the reheating temperature from the considerations of primordial nucleosynthesis and the energy scale of inflation [27.

This work is organized as follows: in Sec. 2 we detail the derivation of the reheating duration and of the temperature at the end of reheating as a function of the spectral index, for canonical single-field inflationary models and for reheating scenarios that can be described in terms of a constant effective equation of state; in Sec. 3 we review the analysis of [28, see also [15], for a power law potential and we discuss the constraints from reheating on the inflationary parameters; in Secs. 4 we compute the relations between inflationary and reheating parameters in the Kähler inflation models and discuss the bounds placed on some of these models by reheating; in Sec. 6] we present our conclusions.

\section{Basic Formalism}

To set up our notation, we briefly review the formalism in [15] and [29] to constrain the the $e$-folding number of reheating $N_{\text {re }}$ and the reheating temperature $T_{\text {re }}$ using an observational constraint on the spectral index $n_{s}$.

For a single-field inflation model with a potential $V(\phi)$, the inflaton obeys

$$
\ddot{\phi}+3 \frac{\dot{a}}{a} \dot{\phi}+\frac{\partial V}{\partial \phi}=0,
$$

where the dot indicates differentiation with respect to cosmic time, and $a$ is the scale factor determined by the Friedman equation:

$$
\left(\frac{\dot{a}}{a}\right)^{2}=\frac{1}{3 M_{\mathrm{pl}}^{2}}\left(\frac{\dot{\phi}^{2}}{2}+V(\phi)\right) .
$$

Adopting the slow-roll approximation during inflation, the above equations are approximated as

$$
\begin{aligned}
& 3 H \dot{\phi}+V^{\prime}(\phi)=0, \\
& H^{2}=\frac{V(\phi)}{3 M_{\mathrm{pl}}^{2}},
\end{aligned}
$$

where the prime denotes differentiation with respect to $\phi$, and $H=\dot{a} / a$ is the Hubble parameter. Using the definitions of the potential slow-roll parameters

$$
\begin{gathered}
\epsilon \equiv \frac{1}{2} M_{\mathrm{pl}}^{2}\left(\frac{V_{\phi}}{V}\right)^{2}, \\
\eta \equiv M_{\mathrm{pl}}^{2}\left(\frac{V_{\phi \phi}}{V}\right)
\end{gathered}
$$

the scalar spectral index is expressed as

$$
n_{s}=1-6 \epsilon+2 \eta
$$

and the tensor-to-scalar ratio as

$$
r_{T}=16 \epsilon
$$

Using the definition of energy density $\left(\dot{\phi}^{2} /\left(2 M_{\mathrm{pl}}^{2}\right)+V(\phi)\right)$ and the slow-roll approximated Eqs. (4) and (5), the energy density during the inflation epoch can be written as $\rho=(1+\epsilon / 3) V$. We take the end of inflation as $\epsilon=1$, at which the energy density of the Universe can be written as

$$
\rho_{\mathrm{end}}=\frac{4}{3} V\left(\phi_{\mathrm{end}}\right)=\frac{4}{3} V_{\mathrm{end}},
$$

where $\phi_{\text {end }}$ is the value of the scalar field at the end of inflation.

Finally the number of e-folds between horizon crossing of a perturbation of wavenumber $k$ and the end of inflation is given by

$$
N_{k} \equiv \ln \left(\frac{a_{\mathrm{end}}}{a_{k}}\right)=-\frac{1}{M_{\mathrm{pl}}^{2}} \int_{\phi_{\text {end }}}^{\phi_{k}}\left(\frac{V}{V_{\phi}}\right) d \phi
$$

where $\phi_{\text {end, }, k}$ are the values of $\phi$ at the end of inflation and when mode $k$ leaves the Hubble radius.

Let us assume that during the reheating epoch, the Universe is dominated by an energy component due to the scalar field with an effective equation-ofstate parameter $w_{\text {re }}$ which is taken to be constant during the entire reheating period. At the end of the reheating epoch, the reheating temperature is related to the energy density of the Universe as

$$
\rho_{\mathrm{re}}=\frac{\pi^{2} g_{\mathrm{re}}}{30} T_{\mathrm{re}}^{4}
$$

where $T_{\text {re }}$ is the reheating temperature, and $g_{\text {re }}$ is the number of relativistic species at the end of reheating, which can be assumed to be $g_{\text {re }}=\mathcal{O}(100)$. 
Defining the scale factor at the end of reheating, $a_{\mathrm{re}}$, we can write the $e$-folding number of the reheating epoch,

$$
\begin{aligned}
N_{\mathrm{re}} \equiv \ln \left(\frac{a_{\mathrm{re}}}{a_{\mathrm{end}}}\right) & = \\
& -\frac{1}{3\left(1+w_{\mathrm{re}}\right)} \ln \left(\frac{\rho_{\mathrm{re}}}{\rho_{\text {end }}}\right),
\end{aligned}
$$

where $a_{\text {re }}$ is the scale factor at the end of reheating.

Now we show how an observational constraint on the spectral index $n_{s}$ can constrain the $e$-folding number $N_{\text {re }}$. This depends on the model, i.e. potential $V(\phi)$, which sometimes (depending upon the expression for potential) makes the analytically writing $N_{\text {re }}$ in terms of $n_{s}$ very difficult. The derivation of $N_{\text {re }}$ hinges on the relation $a_{k} H_{k}=k$, which is satisfied by a perturbation mode with the wavenumber $k$, where $a_{k}$ and $H_{k}$ are respectively the scale factor and Hubble parameter just before the mode $k$ exits Hubble radius during the inflation. In cases where $N_{\text {re }}$ and $n_{s}$ both can be written as function of $\phi_{k}$, a parametric plot can be obtained between $N_{\text {re }}$ and $n_{s}$, otherwise numerical techniques have to applied. Then the logarithmic version of the relation $a_{k} H_{k}=k$ can be broken into different epochs

$$
0=\ln \left(\frac{k}{a_{k} H_{k}}\right)=\ln \left(\frac{a_{\mathrm{end}}}{a_{k}} \frac{a_{\mathrm{re}}}{a_{\mathrm{end}}} \frac{a_{0}}{a_{\mathrm{re}}} \frac{k}{a_{0} H_{k}}\right),
$$

where $a_{0}$ is the scale factor at the present epoch. Using the definitions Eqs. (13) and (11), Eq. (14) yields

$$
N_{k}+N_{\text {re }}+\ln \left(\frac{a_{0}}{a_{\text {re }}}\right)+\ln \left(\frac{k}{a_{0} H_{k}}\right)=0 .
$$

Using the conservation of entropy, we may write

$$
\frac{a_{\mathrm{re}}}{a_{0}}=\left(\frac{43}{11 g_{\text {re }}}\right)^{1 / 3} \frac{T_{0}}{T_{\mathrm{re}}},
$$

where $T_{0}=2.725 \mathrm{~K}$ is the temperature of the Universe at the present epoch. Using Eq. $(12), T_{\text {re }}$ can be replaced with $\rho_{\text {re }}$ in Eq. 16

$$
\frac{a_{\mathrm{re}}}{a_{0}}=\left(\frac{43}{11 g_{r e}}\right)^{1 / 3} T_{0}\left(\frac{\pi^{2} g_{r e}}{30 \rho_{r e}}\right)^{1 / 4} .
$$

However $\rho_{\text {re }}$ can be written in terms of $V_{\text {end }}$ and $N_{\text {re }}$ using connection between them using Eq. (10).
Therefore, using Eqs. (10) and (13), we have

$$
\begin{aligned}
\rho_{\text {re }}=\frac{4}{3} V_{\text {end }}\left(\frac{a_{\mathrm{re}}}{a_{\mathrm{end}}}\right)^{-3\left(1+w_{\mathrm{re}}\right)} & \\
= & \frac{4}{3} V_{\mathrm{end}} e^{-N_{\mathrm{re}} 3\left(1+w_{\mathrm{re}}\right)} .
\end{aligned}
$$

Then, the logarithm of Eq. (17) yields the following expression in terms of $N_{\text {re }}$ :

$$
\begin{aligned}
\ln \left(\frac{a_{\mathrm{re}}}{a_{0}}\right) & =\frac{1}{3} \ln \left(\frac{43}{11 g_{\text {re }}}\right)+\frac{1}{4} \ln \left(\frac{\pi^{2} g_{r e}}{30}\right) \\
+ & \frac{1}{4} \ln \left(\frac{3 T_{0}^{4}}{4 V_{\mathrm{end}}}\right)+\frac{3 N_{\mathrm{re}}\left(1+w_{\mathrm{re}}\right)}{4} .
\end{aligned}
$$

Using the amplitude of the scalar perturbations, $A_{s}=H^{4} /\left(4 \pi^{2} \dot{\phi}^{2}\right)$, and the slow-roll approximation, we may write

$$
H_{k}=\frac{\pi M_{\mathrm{pl}} \sqrt{r A_{s}}}{\sqrt{2}} .
$$

Inserting Eqs. (19) into Eq. (15), we finally have

$$
\begin{gathered}
N_{\mathrm{re}}=\frac{4}{1-3 w_{\mathrm{re}}}\left[-\ln \left(\frac{k}{a_{0} T_{0}}\right)\right. \\
\left.-\frac{1}{4} \ln \left(\frac{30}{\pi^{2} g_{\mathrm{re}}}\right)-\frac{1}{3} \ln \left(\frac{11 g_{\text {re }}}{43}\right)-\ln \left(\frac{V_{\mathrm{end}}^{1 / 4}}{H_{k}}-N_{k}\right)\right] .
\end{gathered}
$$

In our analysis, we use $n_{s}=0.9682 \pm 0.0062$ and $k=0.05 \mathrm{Mpc}^{-1}$ as pivot wavenumber, and $T_{0}=3.522 \times 10^{25} \mathrm{Mpc}^{-1}$ as present temperature of the Universe in the cosmological units. we adopt the amplitude of the scalar perturbation at the pivot scale $A_{s}$ given by $A_{s}=2.196 \times 10^{-9}$ (Table 4 of 30]). Small variations in $A_{s}$ have negligible effects on reheating predictions. We use $n_{s}=0.9682 \pm 0.0062$. The last two terms in Eq. (21) are model dependent. As $g_{\text {re }}$ can be safely taken to be of order of 100 , for a particular wave number, a simple expression for $N_{\text {re }}$ can be obtained for Planck's pivot scale $k=0.05 \mathrm{Mpc}^{-1}$ :

$$
N_{\mathrm{re}}=\frac{4}{1-3 w_{\mathrm{re}}}\left[61.6-\ln \left(\frac{V_{\mathrm{end}}^{1 / 4}}{H_{k}}\right)-N_{k}\right] .
$$

As we will plot $T_{\text {re }}$ on the base-10 logarithmic scale, qualitatively it will be negative scaled version of the

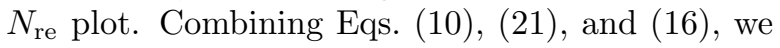
also have 31 .

$$
T_{\text {re }}=\exp \left[-\frac{3}{4}\left(1+w_{\text {re }}\right) N_{\text {re }}\right]\left(\frac{2 V_{\text {end }}}{5 \pi^{2}}\right)^{1 / 4} .
$$


Because the wavenumber $k$ and $n_{s}$ are related implicitly through the scalar field $\phi$, using $H_{k} a_{k}=k$, Eqs. (8) and (11), one can write $N_{\text {re }}$ and $T_{\text {re }}$ as functions of the spectral index $n_{s}$.

In previous works [15, 29] considering constraints on reheating, the analysis has been done only for the pivot scale $k=0.05 \mathrm{Mpc}^{-1}$. Considering $N_{\text {re }}$ as a function of wavenumber $k$, we have extended this to all other observable scales too, particularly to find out what is the maximum possible $N_{\text {re }}$ for each observable scale. To this end, from Eq. (21), $N_{\text {re }}$ becomes a function of $k$ in addition to $n_{s}$. In particular, $V_{\text {end }}$ and $N_{k}$ and $H_{k}$ will be treated as functions of $n_{s}$ through inverting the function $\phi_{k}\left(n_{s}\right)$ whereas dependence on $k$ will arise through the term $\ln \left(\frac{k}{a_{0} T_{0}}\right)$. For pivot scale $k=0.05 \mathrm{Mpc}^{-1}$, the above logarithmic term plus two other model independent terms in Eq. (21) makes 61.6 15. So, in essence, we have generalized previously used $N_{\text {re }}\left(n_{s}\right)_{k=0.05 \mathrm{Mpc}^{-1}}$ to $N_{\text {re }}\left(n_{s}, k\right)$, where to get maximum $N_{\text {re }}, n_{s}$ takes the lower bound or the upper bound value from the constraint on the $n_{s}$, i.e.

$$
\left\{\begin{array}{lll}
\operatorname{Max} N_{\text {re }}\left(n_{s}=0.9620, k\right) & \text { for } & w_{\text {re }}<1 / 3, \\
\operatorname{Max} N_{\text {re }}\left(n_{s}=0.9744, k\right) & \text { for } & w_{\text {re }}>1 / 3 .
\end{array}\right.
$$

From the analytic expression of $N_{\text {re }}$ in Eq. (21), it can be inferred that maximum possible $N_{\text {re }}$ will depend logarithmically on wavenumber $k$, but to get the variation in it with the order of magnitude, we will have to perform numerical calculations for the specific potentials.

\section{Polynomial Potential}

Before moving to the Kähler potential, let us consider a polynomial type potential

$$
V=\frac{1}{2} m^{4-\alpha} \phi^{\alpha}
$$

This was considered in the context of reheating in 28, 32, 33. This model is analytically tractable. The number of e-folds between the time the pivot scale exited the Hubble radius and the end of inflation can be derived using Eq. (11)

$$
N_{k}=\frac{1}{2 \alpha M_{P}^{2}}\left(\phi_{k}^{2}-\phi_{e n d}^{2}\right) \text {. }
$$

The potential in these polynomial models is generally steep enough so that $\phi_{k} \gg \phi_{\text {end }}$, and therefore it is appropriate to approximate

$$
N_{k} \approx \frac{1}{2 \alpha M_{P}^{2}} \phi_{k}^{2}
$$

Notice that the above approximation may not be valid for a general potential. To write $N_{k}$ as a function of $n_{s}$, we observe from Eq. (8) that $n_{s}$ is a function of $\phi$. Therefore by inverting $n_{s}\left(\phi_{k}\right)$ into $\phi_{k}\left(n_{s}\right)$ and using that in Eq. (27) one finds

$$
N_{k}=\frac{\alpha+2}{2\left(1-n_{s}\right)}
$$

From Eq. 20 and using the previous equation, $H_{k}$ is given by 11

$$
H_{k}=\pi M_{P} \sqrt{\frac{4 \alpha A_{s}}{\alpha+2}\left(1-n_{s}\right)} .
$$

If $V_{\text {end }}(\phi)$ is used as such then we have to know the value of the inflaton mass $m$. However owing to the $V \propto \phi^{\alpha}$ (model dependent part) and $V \propto H^{2}$ (from slow-roll Friedmann equation (5)), we can write

$$
V_{\mathrm{end}}(\phi)=3 M_{\mathrm{pl}}^{2} H_{k}^{2} \frac{\phi_{e n d}^{\alpha}}{\phi_{k}}
$$

Lastly one computes $V_{\text {end }}$ in terms of $n_{s}$ and $A_{s}$,

$$
V_{\text {end }}=6 \pi^{2} M_{P}^{4} A_{s} \frac{1}{2^{\alpha / 2}}\left(1-n_{s}\right)\left(\frac{\alpha\left(1-n_{s}\right)}{(\alpha+2)}\right)^{(\alpha+2) / 2}
$$

where the value of the inflaton field at the end of inflation $\phi_{\text {end }}$ follows from the condition $\epsilon=1$.

Thus $N_{k}, H_{k}$, and $V_{\text {end }}$ are all expressed as functions only of $\alpha, n_{s}$ and $A_{s}$ and one may plot $N_{r e}$ (and $T_{r e}$ ) as a function of $n_{s}$ for some fixed values of $w_{r e}$ and $\alpha$.

\footnotetext{
1 There were typographical errors in Eqs.(3.5) and (3.6) of Ref. 15], which have been corrected here.
} 


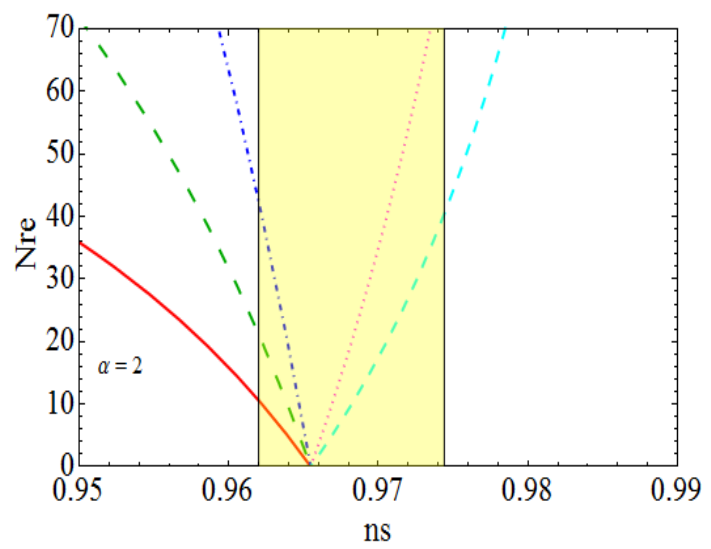

(a)

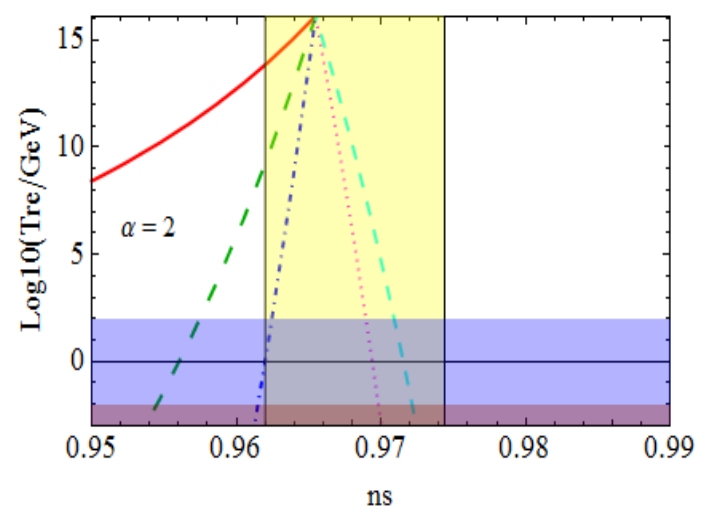

(b)

Fig. 1: (color online). Plots of $N_{r e}$ and $T_{r e}$, the length of reheating and the temperature at the end of reheating respectively, for polynomial potentials with exponent $\alpha$. The solid red line corresponds to $w_{r e}=-1 / 3$, the dashed green line to $w_{r e}=0$, the dot-dashed blue line to $w_{r e}=1 / 6$, the dotted magenta line to $w_{r e}=2 / 3$ and the dot-dashed black line to $w_{r e}=1$. The yellow shaded region corresponds to the $1 \sigma$ bounds on $n_{s}$ from Planck. In (b) temperatures below the light purple shaded region are ruled out by BBN. The light purple pink region is below the electroweak scale, assumed $100 \mathrm{GeV}$ for reference. This region is not disallowed but would be interesting in the context of baryogenesis.

We take instantaneous reheating as the limit $N_{r e} \rightarrow 0$, visualized in the $N_{r e}$ and $T_{\text {re }}$ plots as the point where all the $w_{r e}$ lines meet. Such instantaneous reheating leads to the maximum temperature at the end of reheating, and the equation of state parameter $w_{r e}$ is irrelevant. We plot in Fig. $11 N_{r e}$ and $T_{r e}$ predictions for $\alpha=2$ and 3. As the instantaneous reheating is lying near the central value of $n_{s}, \alpha=2$ give promising predictions when compared with the Planck data. As explained in Ref. [15], for $\alpha=2 / 3$, our polynomial potential takes the form which is favored by axion-monodromy models. As the instantaneous reheating point comes out of the yellow strip in Fig. 2, the cases $\alpha=3$ and $\alpha=4$ (not plotted for the reason of space) are difficult to reconcile with $w_{r e} \leq 1$ even if one considers the $2 \sigma$ bounds on $n_{s}{ }^{2}$ 


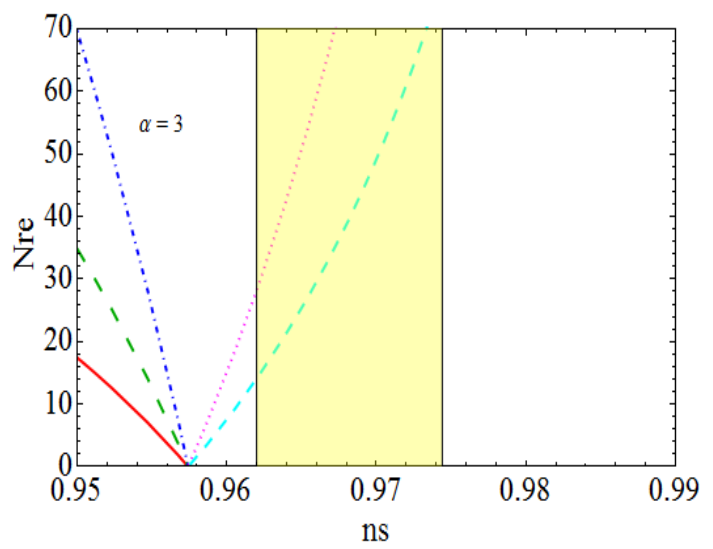

(a)

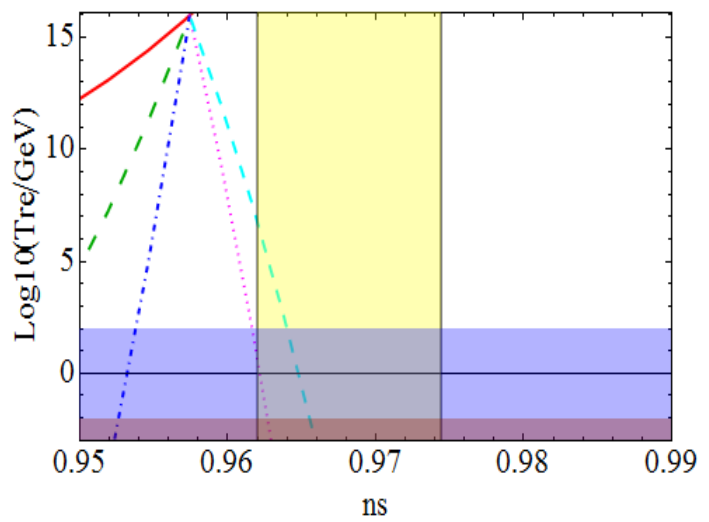

(b)

Fig. 2: (color online). Same as for figure 1 but here we have taken $\alpha=3$.

\section{Kähler Potential}

These models are string inspired models and appear in type IIB string theories from Calabi-Yau compactification. KMII scenarios have been derived and studied in 36 41. If internal spaces are weighted projective spaces, one of the Kähler moduli can be considered as an inflaton field and its potential, in the large field limit, reads

$$
V(\phi)=M^{4}\left(1-\alpha \frac{\phi}{M_{\mathrm{Pl}}} e^{-\phi / M_{\mathrm{P} 1}}\right),
$$

where $\alpha$ is a positive dimensionless parameter.

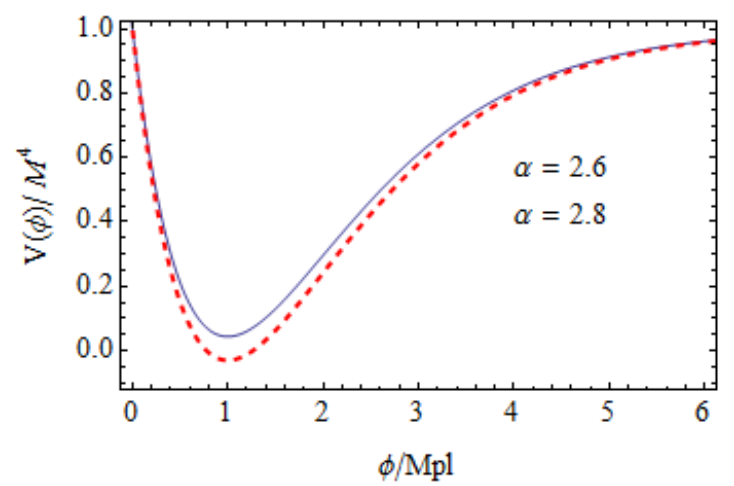

Fig. 3: An illustration of the potential for different values of $\alpha$.

This potential depends on one free parameter, $\alpha$. As explained in 32, in order for slow-roll inflation to occur, the range of possible values for $\alpha$ is restricted. Within the narrow allowed range, the predictions of the model is almost independent of $\alpha$. The potential (32) for two values of $\alpha$ is displayed in Fig. 3. The potential blows up at $\phi=0$, and then decreases as $\phi$ increases. It reaches a minimum at $\phi=M_{\mathrm{Pl}}$. Note that the minimum is lower for $\alpha=2.8$ than for $\alpha=2.6$. The asymptotic value $V=M^{4}$ is reached when $\phi \rightarrow+\infty$. As originating from the string theory, the potential is derived under the large field assumption. Therefore inflation is assumed to proceed from the right to the left, in the increasing $\phi>M_{\mathrm{Pl}}$ branch of the potential. It is to be observed that the minimum value of the potential is located at $\phi=M_{\mathrm{Pl}}$ and is $V_{\min }=M^{4}(1-\alpha / e)$. Therefore, for the potential to be positive definite everywhere, the condition $0<\alpha<e \simeq 2.72$ must be satisfied. However, this condition is relevant only when one considers both the increasing $\left(\phi>M_{\mathrm{Pl}}\right)$ as well as decreasing $\left(\phi<M_{\mathrm{Pl}}\right)$ branch of the potential, and can be safely ignored if one restricts the field excursion to the increasing branch only.

\subsection{Slow-roll parameters}

The slow-roll parameters for the Käher potential can be expressed as

$$
\epsilon(\phi)=\frac{\alpha^{2}\left(M_{\mathrm{Pl}}-\phi\right)^{2}}{2\left(M_{\mathrm{Pl}} e^{\frac{\phi}{M_{\mathrm{P} 1}}}-\alpha \phi\right)^{2}},
$$




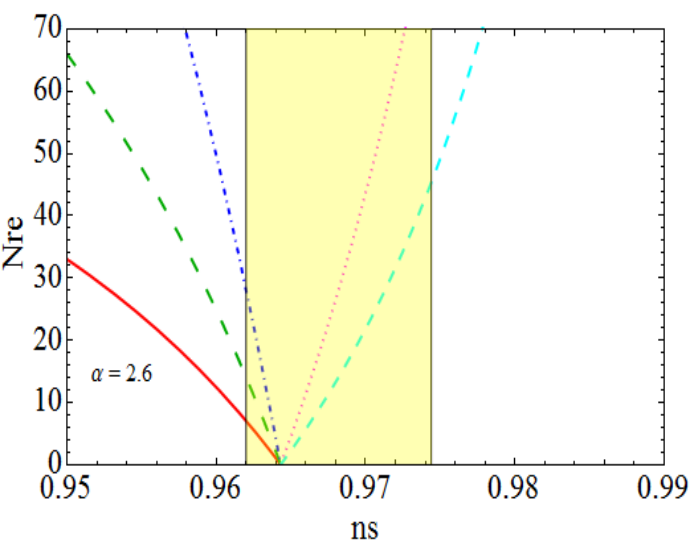

(a)

$$
\eta(\phi)=\frac{\alpha\left(\phi-2 M_{\mathrm{Pl}}\right)}{\alpha \phi-M_{\mathrm{Pl}} e^{\frac{\phi}{M_{\mathrm{Pl}}}}},
$$

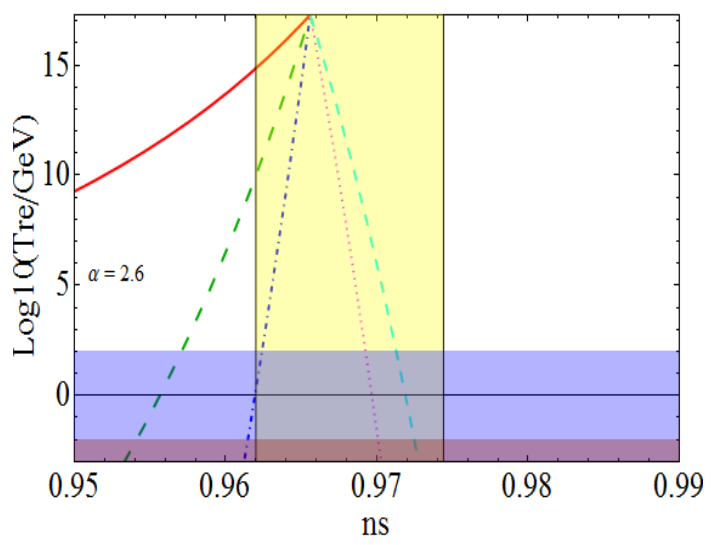

(b)

and therefore using (8), we get

Fig. 4: (color online). Plots of $N_{\text {re }}$ and $T_{\text {re }}$, the length of reheating and the temperature at the end of reheating respectively, for Kähler potential with exponent $\alpha=2.6$.

$n_{s}=\frac{M_{\mathrm{Pl}}\left(\alpha^{2}\left(2 \phi-3 M_{\mathrm{Pl}}\right)+4 \alpha e^{\frac{\phi}{M_{\mathrm{Pl}}}}\left(M_{\mathrm{Pl}}-\phi\right)+M_{\mathrm{Pl}} e^{\frac{2 \phi}{M_{\mathrm{Pl}}}}\right)}{\left(M_{\mathrm{Pl}} e^{\frac{\phi}{M_{\mathrm{Pl}}}}-\alpha \phi\right)^{2}}$.

As $n_{s}$ depends on $\phi$ in a complex way and can not be inverted to get $\phi\left(n_{s}\right)$, we will have to do numerical simulation, whose results are shown in Figs. 4 to 5 . 


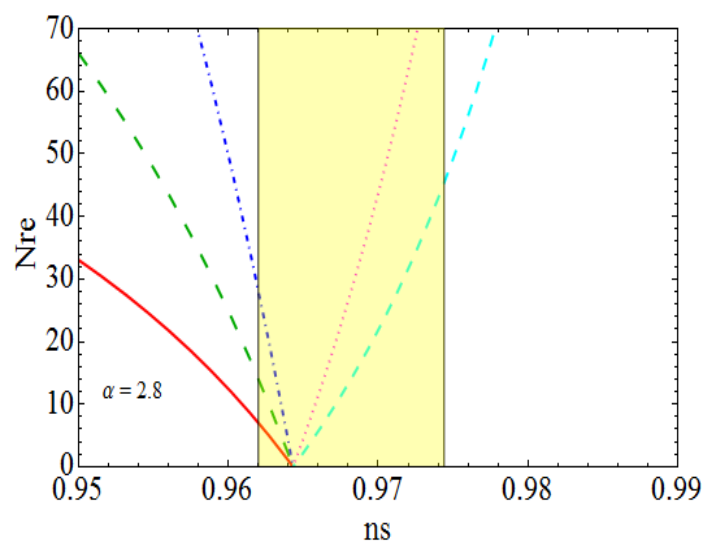

(a)

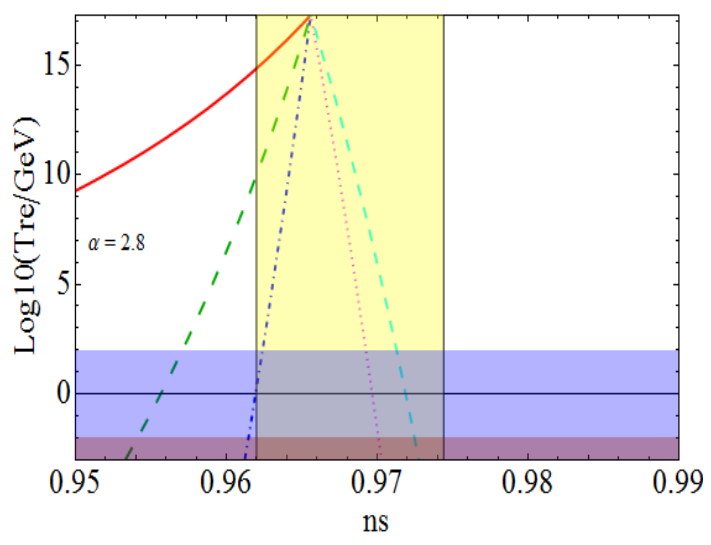

(b)

Fig. 5: (color online). Same as for figure 4 but here we have taken $\alpha=2.8$.

For $w_{\mathrm{re}}=1 / 3$,assuming $g_{r e}=100$, and Planck's pivot scale, Eq. 21] implies

$$
61.6=\ln \left(\frac{V_{\text {end }}^{\frac{1}{4}}}{H_{k}}\right)+N_{k} .
$$

Using Planck's central value for $A_{s}$, Eq. 36 gives specific predictions for $n_{s}$

$$
\begin{cases}n_{s}=0.964225 & \text { for } \quad \alpha=2.6, \\ n_{s}=0.964250 & \text { for } \quad \alpha=2.7, \\ n_{s}=0.965277 & \text { for } \quad \alpha=2.8 .\end{cases}
$$

Fig 6 shows the variation of maximum $N_{\text {re }}$ versus wave number $k$ for fixed $w_{\text {re }}$ and $\alpha$. As already pointed in Sec. 2, the variation is logarithmic in nature and the effect of $w_{\text {re }}$ is that of scaling the $\mathrm{y}$-axis. For a fixed $w_{\text {re }}$, there was minor variation in the plots for $\alpha=2.8$ and $\alpha=2.6$, obviously due to minor horizontal variation in the convergent point for the potential(see Fig. 4 and 5 .

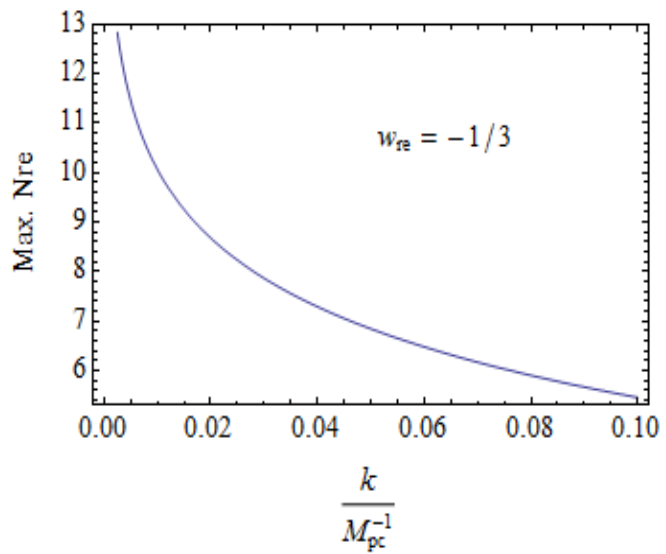

(a)

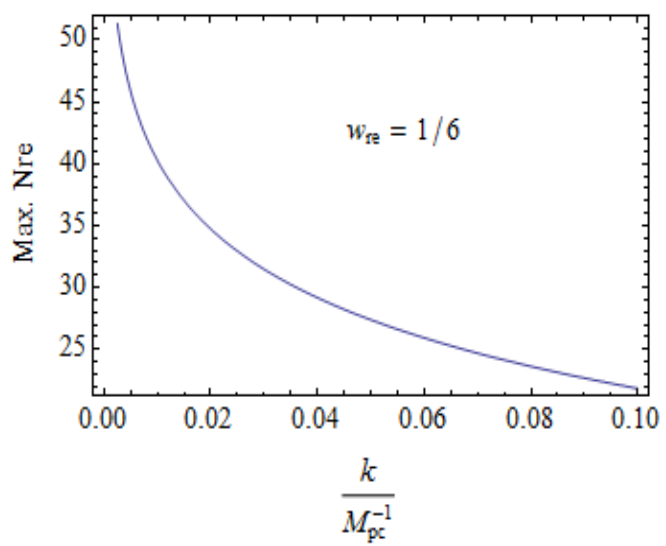

(b)

Fig. 6: (color online). For $\alpha=2.8$, the variation in

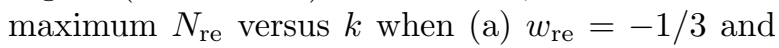
$w_{\text {re }}=1 / 6$, respectively.

\section{Discussion of Results}

As is apparent from the plots of $N_{r e}$ and $T_{\text {re }}$ in [15, 29] and the present work for different potentials, qualitatively the $w_{r e}$ curves are same for different potentials due to slow-roll approximation. 
In other words we can say that slow-roll approximation overrides the differences in potential shapes for $N_{r e}$ (or $T_{\text {re }}$ ) vs $n_{s}$ curves. These characteristic curves are due to the solution being on the slow-roll trajectory. Further, we make few general observations on the $N_{r e}$ and $T_{\text {re }}$ plots. The role of single parameter $\alpha$ is just to shift the group of $w_{r e}$ curves horizontally, whereas each $w_{r e}$ curves are tilted to the right or the left depending upon whether $w_{r e}$ is less or greater than $\frac{1}{3}$, respectively. Apparently, a $w_{r e}=\frac{1}{3}$ (not shown) curve would correspond to a vertical line which passes through the instantaneous reheat point [15. It is also to be noticed that in all the potentials treated within this formalism, the $T_{\text {re }}$ converges around $10^{16} \mathrm{GeV}[15,28,29$.

\section{Summary and Conclusion}

In this Letter, we have analyzed the reheating constraint on the Kähler moduli inflation within the slow-roll regime, following the formalism as in $[15,28,29$. Although there is no new observational constraint introduced in this reheating analysis as compared to ones trying to constrain standard inflation using $\left(n_{s}, r_{T}\right)$ plots 42, embedding the eos parameter has enriched this analysis. That is the reason why this analysis can further constrain the allowed inflationary models. However as already stated, the details of single parameter potential, in general, are irrelevant if the analysis is done strictly within the slow-roll approximation.

\section{Acknowledgments}

RK thanks Jessica Cook for useful communication.

\section{References}

[1] P. A. R. Ade, et al., Planck 2013 results. XXII. Constraints on inflation arXiv: 1303.5082

[2] P. A. R. Ade, et al., Planck 2013 results. XXIV. constraints on primordial non-gaussianity, arXiv:1303.5084 [astro-ph] URL http://arxiv.org/abs/1303.5084

[3] L. Kofman, The Origin of matter in the universe: Reheating after inflation, arXiv preprint astroph/9605155. URL http://arxiv.org/abs/astro-ph/9605155

[4] M. A. Amin, M. P. Hertzberg, D. I. Kaiser, J. Karouby, Nonperturbative Dynamics Of Reheating After Inflation: A Review, International Journal of Modern Physics D 24 (01) (2015) 1530003, arXiv: 1410.3808. doi:10.1142/S0218271815300037 URL http://arxiv.org/abs/1410.3808
[5] L. Kofman, A. Linde, A. Starobinsky, Reheating after Inflation Physical Review Letters 73 (24) (1994) 3195-3198, arXiv: hep-th/9405187. doi:10.1103/ PhysRevLett.73.3195

URL http://arxiv.org/abs/hep-th/9405187

[6] L. Kofman, A. Linde, A. Starobinsky, Towards the Theory of Reheating After Inflation Physical Review D 56 (6) (1997) 3258-3295, arXiv: hep-ph/9704452. doi:10.1103/PhysRevD.56.3258

URL http://arxiv.org/abs/hep-ph/9704452

7] B. R. Greene, T. Prokopec, T. G. Roos, Inflaton Decay and Heavy Particle Production with Negative Coupling. Physical Review D 56 (10) (1997) 6484-6507, arXiv: hep-ph/9705357. doi:10.1103/PhysRevD.56.6484 URL http://arxiv.org/abs/hep-ph/9705357

[8] N. Shuhmaher, R. Brandenberger, Non-Perturbative Instabilities as a Solution of the Cosmological Moduli Problem, Physical Review D 73 (4), arXiv: hepth/0507103. doi:10.1103/PhysRevD.73.043519 URL http://arxiv.org/abs/hep-th/0507103

[9] J. F. Dufaux, G. N. Felder, L. Kofman, M. Peloso, D. Podolsky, Preheating with Trilinear Interactions: Tachyonic Resonance Journal of Cosmology and Astroparticle Physics 2006 (07) (2006) 006-006, arXiv: hep-ph/0602144. doi:10.1088/1475-7516/2006/07/ 006.

URL http://arxiv.org/abs/hep-ph/0602144

[10] A. A. Abolhasani, H. Firouzjahi, M. M. Sheikh-Jabbari, Tachyonic Resonance Preheating in Expanding Universe Physical Review D 81 (4), arXiv: 0912.1021. doi:10.1103/PhysRevD.81.043524 URL http://arxiv.org/abs/0912.1021

[11] G. Felder, L. Kofman, A. Linde, Tachyonic Instability and Dynamics of Spontaneous Symmetry Breaking. Physical Review D 64 (12), arXiv: hep-th/0106179. doi:10.1103/PhysRevD.64.123517

URL http://arxiv.org/abs/hep-th/0106179

[12] G. Felder, L. Kofman, A. Linde, Instant Preheating, Physical Review D 59 (12), arXiv: hep-ph/9812289. doi:10.1103/PhysRevD.59.123523 URL http://arxiv.org/abs/hep-ph/9812289

[13] R. C. de Freitas, S. V. B. Gonalves, CMB Constraints on Reheating Models with Varying Equation of State. arXiv:1509.08500 [astro-ph]ArXiv: 1509.08500 . URL http://arxiv.org/abs/1509.08500

[14] K. D. Lozanov, M. A. Amin, The Equation of State and Duration to Radiation Domination After Inflation arXiv:1608.01213 [astro-ph, physics:hep-ph, physics:hep-th]ArXiv: 1608.01213. URL http://arxiv.org/abs/1608.01213

[15] J. L. Cook, E. Dimastrogiovanni, D. A. Easson, L. M. Krauss, Reheating predictions in single field inflation Journal of Cosmology and Astroparticle Physics 2015 (04) (2015) 047-047, arXiv: 1502.04673. doi: 10.1088/1475-7516/2015/04/047 URL http://arxiv.org/abs/1502.04673

[16] A. Taruya, Y. Nambu, Cosmological perturbation with two scalar fields in reheating after inflation Physics Letters B 428 (1-2) (1998) 37-43, arXiv: gr-qc/9709035. doi:10.1016/S0370-2693(98)00378-5 URL http://arxiv.org/abs/gr-qc/9709035

[17] B. A. Bassett, F. Viniegra, Massless Metric Preheating Physical Review D 62 (4), arXiv: hep-ph/9909353. doi: 10.1103/PhysRevD.62.043507. URL http://arxiv.org/abs/hep-ph/9909353 
[18] F. Finelli, R. Brandenberger, Parametric Amplification of Metric Fluctuations During Reheating in Two Field Models, Physical Review D 62 (8), arXiv: hepph/0003172. doi:10.1103/PhysRevD.62.083502 URL http://arxiv .org/abs/hep-ph/0003172

[19] S. Tsujikawa, B. A. Bassett, When can preheating affect the CMB? Physics Letters B 536 (1-2) (2002) 9-17, arXiv: astro-ph/0204031. doi:10.1016/ S0370-2693(02)01813-0.

URL http://arxiv.org/abs/astro-ph/0204031

[20] A. Chambers, A. Rajantie, Lattice calculation of nonGaussianity from preheating, Physical Review Letters 100 (4), arXiv: 0710.4133. doi:10.1103/PhysRevLett. 100.041302

URL http://arxiv.org/abs/0710.4133

[21] J. R. Bond, A. V. Frolov, Z. Huang, L. Kofman, NonGaussian Spikes from Chaotic Billiards in Inflation Preheating Physical Review Letters 103 (7), arXiv: 0903.3407. doi:10.1103/PhysRevLett.103.071301 URL http://arxiv.org/abs/0903.3407

[22] B. A. Bassett, D. I. Kaiser, R. Maartens, General Relativistic effects in preheating, Physics Letters B 455 (14) (1999) 84-89, arXiv: hep-ph/9808404. doi : 10.1016/ S0370-2693(99) 00478-5.

URL http://arxiv.org/abs/hep-ph/9808404

[23] B. A. Bassett, F. Tamburini, D. I. Kaiser, R. Maartens, Metric preheating and limitations of linearized gravity Nuclear Physics B 561 (1-2) (1999) 188-240, arXiv: hep-ph/9901319. doi:10.1016/S0550-3213(99) 00495-2 URL http: //arxiv.org/abs/hep-ph/9901319

[24] B. A. Bassett, C. Gordon, R. Maartens, D. I. Kaiser, Restoring the sting to metric preheating Physical Review D 61 (6), arXiv: hep-ph/9909482. doi:10.1103/ PhysRevD.61.061302

URL http://arxiv.org/abs/hep-ph/9909482

[25] L. Bethke, D. G. Figueroa, A. Rajantie, Anisotropies in the Gravitational Wave Background from Preheating, Physical Review Letters 111 (1), arXiv: 1304.2657. doi:10.1103/PhysRevLett.111.011301 URL http://arxiv.org/abs/1304.2657

[26] R. Easther, R. Galvez, O. Ozsoy, S. Watson, Supersymmetry, Nonthermal Dark Matter and Precision Cosmology 1 Physical Review D 89 (2), arXiv: 1307.2453. doi:10.1103/PhysRevD.89.023522

URL http://arxiv.org/abs/1307.2453

[27] G. Steigman, Primordial Nucleosynthesis in the Precision Cosmology Era Annual Review of Nuclear and Particle Science 57 (1) (2007) 463-491, arXiv: 0712.1100. doi:10.1146/annurev.nucl.56.080805. 140437 .

URL http://arxiv.org/abs/0712.1100

[28] L. Dai, M. Kamionkowski, J. Wang, Reheating constraints to inflationary models, Physical Review Letters 113 (4), arXiv: 1404.6704. doi:10.1103/PhysRevLett. 113.041302

URL http://arxiv .org/abs/1404.6704

[29] Y. Ueno, K. Yamamoto, Constraints on $\backslash$ textbackslashalpha-attractor inflation and reheating, Physical Review D 93 (8), arXiv: 1602.07427. doi:10.1103/PhysRevD.93.083524.

URL http://arxiv.org/abs/1602.07427

[30] P. A. R. Ade, et al., Planck 2015 results. XIII. Cosmological parameters, arXiv:1502.01589 [astro-ph]ArXiv: 1502.01589 .
URL http://arxiv.org/abs/1502.01589

[31] M. Eshaghi, M. Zarei, N. Riazi, A. Kiasatpour, CMB and reheating constraints to \alpha-attractor inflationary models Physical Review D 93 (12), arXiv: 1602.07914. doi:10.1103/PhysRevD.93.123517 URL http://arxiv.org/abs/1602.07914

[32] J. Martin, C. Ringeval, V. Vennin, Encyclopaedia Inflationaris, arXiv:1303.3787 [astro-ph, physics:gr-qc, physics:hep-ph, physics:hep-th]ArXiv: 1303.3787. URL http://arxiv.org/abs/1303.3787

[33] J. Martin, C. Ringeval, V. Vennin, Observing the Inflationary Reheating Physical Review Letters 114 (8), arXiv: 1410.7958. doi:10.1103/PhysRevLett.114. 081303 URL http://arxiv.org/abs/1410.7958

[34] S. Bartrum, M. Bastero-Gil, A. Berera, R. Cerezo, R. O. Ramos, J. G. Rosa, The importance of being warm (during inflation) Physics Letters B 732 (2014) 116121, arXiv: 1307.5868. doi:10.1016/j.physletb. 2014 . 03.029

URL http://arxiv.org/abs/1307.5868

[35] M. Bastero-Gil, A. Berera, R. O. Ramos, J. G. Rosa, Observational implications of mattergenesis during inflation Journal of Cosmology and Astroparticle Physics 2014 (10) (2014) 053-053, arXiv: 1404.4976. doi: 10.1088/1475-7516/2014/10/053 URL http://arxiv.org/abs/1404.4976

[36] J. P. Conlon, F. Quevedo, Kahler Moduli Inflation, Journal of High Energy Physics 2006 (01) (2006) 146146, arXiv: hep-th/0509012. doi:10.1088/1126-6708/ 2006/01/146

URL http://arxiv.org/abs/hep-th/0509012

[37] J. R. Bond, L. Kofman, S. Prokushkin, P. M. Vaudrevange, Roulette Inflation with K\"ahler Moduli and their Axions Physical Review D 75 (12), arXiv: hepth/0612197. doi:10.1103/PhysRevD.75.123511 URL http://arxiv.org/abs/hep-th/0612197

[38] S. Krippendorf, F. Quevedo, Metastable SUSY Breaking, de Sitter Moduli Stabilisation and K\" ahler Moduli Inflation Journal of High Energy Physics 2009 (11) (2009) 039-039, arXiv: 0901.0683. doi:10.1088/ 1126-6708/2009/11/039

URL http://arxiv.org/abs/0901.0683

[39] J. J. Blanco-Pillado, D. Buck, E. J. Copeland, M. Gomez-Reino, N. J. Nunes, Kahler Moduli Inflation Revisited Journal of High Energy Physics 2010 (1), arXiv: 0906.3711. doi:10.1007/JHEP01(2010)081. URL http://arxiv.org/abs/0906.3711

[40] M. Kawasaki, K. Miyamoto, Kahler moduli double inflation Journal of Cosmology and Astroparticle Physics 2011 (02) (2011) 004-004, arXiv: 1010.3095. doi: 10.1088/1475-7516/2011/02/004 URL http://arxiv.org/abs/1010.3095

[41] S. Lee, S. Nam, $\mid \mathrm{K} \backslash$ "\{a\}hler moduli inflation and WMAP7, International Journal of Modern Physics A 26 (06) (2011) 1073-1096, arXiv: 1006.2876. doi: 10.1142/S0217751X1105155X URL http://arxiv.org/abs/1006.2876

[42] R. Kabir, A. Mukherjee, D. Lohiya, Reanalyzing the upper limit on the tensor-to-scalar perturbation ratio r_t in a quartic potential inflationary model Physics Letters B 736 (2014) 246-251, arXiv: 1405.5136. doi: $10.1016 / j \cdot$ physletb.2014.07.034 URL http://arxiv.org/abs/1405.5136 